\title{
Zika Virus: A Review of Management Considerations and Controversies at Six Months
}

\author{
Laura Singer, MPH; Kelly G. Vest, DVM, MPH, DrPH; Charles W. Beadling, MD
}

\section{ABSTRACT}

Zika virus continues to pose a significant global health threat. While the outbreak pattern may seemingly mirror those of other arboviruses, unique transmission characteristics and clinical outcomes warrant a different approach to traditional public health practices. Sexual transmission and virus-associated fetal and nonfetal neurologic disorders specifically challenge conventional methods of disease protection and prevention with regard to vector control, disease surveillance, and health risk communication. The protocols for outbreak and case limitation led by the World Health Organization (in accordance with Public Health Emergency of International Concern declaration) may be augmented by localized risk categorization and assignment for Zika and future emergent outbreaks. There is currently a great deal of "behind the scenes" discussion about modifications to the formal process described in the International Health Regulations. A scalable, adaptable, and flexible process is needed that can be customized to a specific threat. (Disaster Med Public Health Preparedness. 2017;11:279-284)

Key Words: risk, surveillance, transmission, vector, Zika virus

$\mathrm{D}$ r. Frederick Burkle recently stated in an editorial, "Each pandemic serves as a real world exercise on how to best participate and generate the global debate on decisions to protect the global good." So it is with Zika virus (ZIKV). On 1 February 2016, the World Health Organization (WHO) issued a Public Health Emergency of International Concern (PHEIC) announcement related to an initial cluster of microcephaly cases in Brazil and other neurological disorders reported in French Polynesia. ${ }^{2}$ Since that time, additional published research has provided evidence linking maternal exposure to ZIKV to congenital and fetal/neonatal developmental disorders and other neurologic disorders including Guillain-Barré Syndrome. ${ }^{3-5}$

Endemic and epidemic diseases similar to ZIKV (ie, West Nile and dengue viruses), although globally recognized and widespread, have not caused the same alarming concern. Initial infections from all 3 pathogens are usually asymptomatic or have mild symptoms; West Nile fever can be severe, with encephalitis or meningitis, and severe dengue can result in sometimes fatal hemorrhagic fever. However, while infections with ZIKV are generally less dramatic than those with dengue or West Nile viruses, ZIKV is remarkable for the congenital nervous system sequelae in the children of infected pregnant women.

Other newly emergent diseases, such as Ebola, SARS, Legionnaire's disease, and MERS-CoV, have also not evoked the kind of emotional response that ZIKV has.
One could argue that humans are hard-wired as a species to protect our young. The fetal and neonatal defects causing mortality or long-lasting morbidity place a new perspective on the public health management of emergent diseases, raising the stakes and creating challenges to standard public health preparedness and response algorithms.

\section{A GLOBAL EVENT}

A PHEIC declaration, in accordance with the WHO's International Health Regulations, ${ }^{6}$ places emphasis on the severity of a worsening global health situation to advance timelines and mechanisms for in-country and international response. ${ }^{7}$ Declarations are accompanied by an inclusive protocol, customizable depending on the specific "event" and affected countries. "Advance[d] timelines and mechanisms for response" in the case of ZIKV, however, are highly challenging to operationalize in the face of a disease that is accompanied by a long list of unknowns.

Furthermore, while response measures are crucial at this time with regard to influencing the global spread of ZIKV, the fact that the virus is poorly understood and unpredictable warrants paying increased attention to other components of the disaster management cycle (ie, prevention, protection, preparedness, and mitigation), as it seems high-risk to assume that a vaccine will be soon available. ${ }^{8,9}$ Given the aforementioned characteristics of the ZIKV outbreak, one could argue that good public health policy demands that a number of key activities be 
in practice in some form in both affected and unaffected countries (that may be at risk of virus and/or vector importation). This is especially important in light of mass gatherings hosted in Brazil, the epicenter of the ongoing ZIKV outbreak.

Increasing pressure is on Brazil, internally and internationally, as the host of the 2016 World Olympics and Paralympic Games. Ironically, the ZIKV outbreak began in Brazil likely as the result of an imported transmission during an international sports event in 2014. ${ }^{10}$ Such mass gathering events can serve as vehicles for rapidly dispersing diseases from one country to another. Yet the WHO assesses that cancelling or changing the location of the Olympic games "will not significantly alter international spread of Zika virus." 11 Similarly, the study performed by the Centers for Disease Control and Prevention corroborates this sentiment based on other modeling approaches to the problem. ${ }^{12}$

\section{FOCUS ON THE VECTOR}

It is well understood that disease transmission is interrupted or reduced by removing the vector in a vector-borne disease. The spread of ZIKV is not surprising after witnessing the spread of chikungunya virus throughout the Western Hemisphere. ${ }^{13,14}$ What should be surprising is the fact that dengue is endemic in the same geographic areas as ZIKV and has been for decades. Any existing vector control for dengue should have protected against the spread of ZIKV; however, this is not what has been observed. Perhaps either the virus or the vector has mutated and can no longer be controlled by standard vector eradication methods, or there is a lack of national or local governmental support to confront the complex issue of vector control.

Aedes aegypti and Aedes albopictus mosquitoes are resilient and adaptive and prefer to live near their hosts (humans). During the 1950s and 1960s, A. aegypti mosquitoes were nearly eradicated from the Western Hemisphere, yet a small nidus of mosquitoes remained. Along with the importation of A. albopictus during the 1980s, this served to seed North, Central, and South America. ${ }^{15-17}$ Since the mosquito is urbanized, many blame communities and individual behaviors (especially those contributing to standing water) for the persistent company kept with the mosquito. Yet, effective control measures require continued buy-in and financial support, primarily of key government leaders and ministries.

Until the onslaught of media attention, this response has been greatly lacking in the affected countries and many countries with competent vectors. It is likely that fear of impacting trade and travel has compromised any tendency to aggressively lean forward in managing ZIKV. Many experts now believe that both a top-down and a bottom-up approach involving a fully integrated pest management method (see the text box) is necessary to effectively control ZIKV vectors. ${ }^{15,16}$

Unfortunately, once the disease has been introduced into an environment, it is substantially more challenging to implement
Integrated pest management mosquito (Aedes spp.) control includes the combined incorporation of techniques such as habitat control (community, environment), chemical control (focused application of insecticides, adulticides, larvicides), biological control (Bacillus thuringiensis israelensis; Wolbachia), and use of larvivorous fish and genetically modified mosquitoes to affect and influence all of the mosquito lifecycle stages.

prevention and preparedness measures to mitigate disease transmission and spread. Dr. Jeffrey Duchin emphasized this point in a recent commentary by stating, "[i]t is difficult, if not impossible, to develop needed systems, resources and response capacity in the midst of a crisis." ${ }^{18}$ To have greater preventive impact, government leaders and agencies will need to consider a greater investment in vector control and disease surveillance capacity. The fact that the disease in Brazil is estimated to have emerged in 2014 and remained undetected for nearly a full year illustrates the challenge of effective surveillance. ${ }^{10}$

Another ZIKV complication is the fact that it shares both the same vector and clinical presentation as dengue and chikungunya. Theoretically, patients may be co-infected with 2 or all 3 of the diseases at the same time. New evidence suggests that prior dengue infection may accentuate ZIKV clinical severity through antibody-dependent enhancement. ${ }^{19}$ In resource-limited settings, it is predictable that the ability to provide definitive lab diagnosis to distinguish between the diseases can be delayed, and the capacity to provide supportive care in severe cases will be inadequate.

Exposure to ZIKV is primarily based on the risk of being bitten by an infected mosquito. Nevertheless, ZIKV is again different from other arboviruses because transmission can occur sexually. As such, individuals who live in, travel to, or work in active transmission areas will be required to make consequential decisions when considering protection and prevention. Specifically, and unlike in the instances of dengue or chikungunya, women and men alike must identify the needs of their partner, their spouse, and if they are of child-bearing age, their future children. Even though most infections are asymptomatic, the risk of infection must be weighed against the chances of getting pregnant (for women) and/or transmitting the disease to a partner (for both men and women). ${ }^{20,21}$

The idea of changing one's behavior to use vector avoidance as well as consider sexual protective measures or abstinence is new for a vector-borne disease. Risk communication will require carefully tailored messaging for travelers, patients, and parents. Clearly, travelers to endemic or epidemic areas will need to be educated differently than natives, and pregnant women will require more specific attention. In areas where serologic testing is inconclusive or unavailable for ZIKV or dengue, patients may need to be clinically managed for both infections. ${ }^{22}$ For pregnant patients with suspected ZIKV infection, health care providers providing prenatal care must be prepared to diagnose and address fetal developmental complications including death. 


\section{ZIKA VIRUS INFECTION SURVEILLANCE}

With the PHEIC declaration of neurologic complications associated with ZIKV infection, the WHO will follow its surveillance algorithm in accordance with International Health Regulations protocol and procedures. ${ }^{23}$ Disease surveillance in the context of ZIKV and associated congenital and other neurologic disorders has proven far more complex than simple measures of clinical case incidence and distribution. The critical nature of enhanced surveillance practices is exemplified by emerging scientific developments. For example, a 29 June 2016 study observed that $20 \%$ of neurologically affected babies were normocephalic. ${ }^{24}$ Thus, it may be necessary to surveil for more than ZIKV infection and microcephaly; at least in Brazil, screening of apparently healthy normocephalic newborns of every suspected infected pregnant woman may reveal troubling truths.

In the WHO interim guidance for ZIKV infection surveillance, 4 "context" categories associated with "risk of occurrence of severe complications or sequelae associated with Zika virus infection" are identified. ${ }^{25}$ Along with these risk categories, guidance was provided on disease surveillance (Table 1) as well as on reporting requirements (Table 2) based on the transmission risk categories and disease

\section{TABLE 1}

\section{Recommended World Health Organization Zika Virus Surveillance Activities Based on Risk Category ${ }^{\mathrm{a}}$}

\begin{tabular}{|c|c|c|c|c|}
\hline Surveillance Activities $^{c}$ & \multicolumn{4}{|c|}{ Risk Categories $^{b}$} \\
\hline Containment measures and vector control & & $X$ & $X$ & $x$ \\
\hline Detect autochthonous transmission & & $x$ & & \\
\hline Detect changing transmission patterns & & & $x$ & \\
\hline $\begin{array}{l}\text { Identify disease demographics and } \\
\text { epidemiology }\end{array}$ & & & $x$ & $x$ \\
\hline Identify severe complications & & & $x$ & $X$ \\
\hline Investigate past circulation & & $x$ & $X$ & \\
\hline Monitor geographic spread and distribution & & & $x$ & $x$ \\
\hline
\end{tabular}

${ }^{a}$ Adapted from the World Health Organization Surveillance for Zika virus infection, microcephaly and Guillain-Barre syndrome: Interim Guidance (April 7, 2016). ${ }^{25}$

borld Health Organization Zika virus surveillance risk categories.

${ }^{\mathrm{c}}$ Recommended surveillance activities.

\section{TABLE 2}

\section{Recommended World Health Organization Zika Virus Reporting Requirements Based on Risk Categorya}

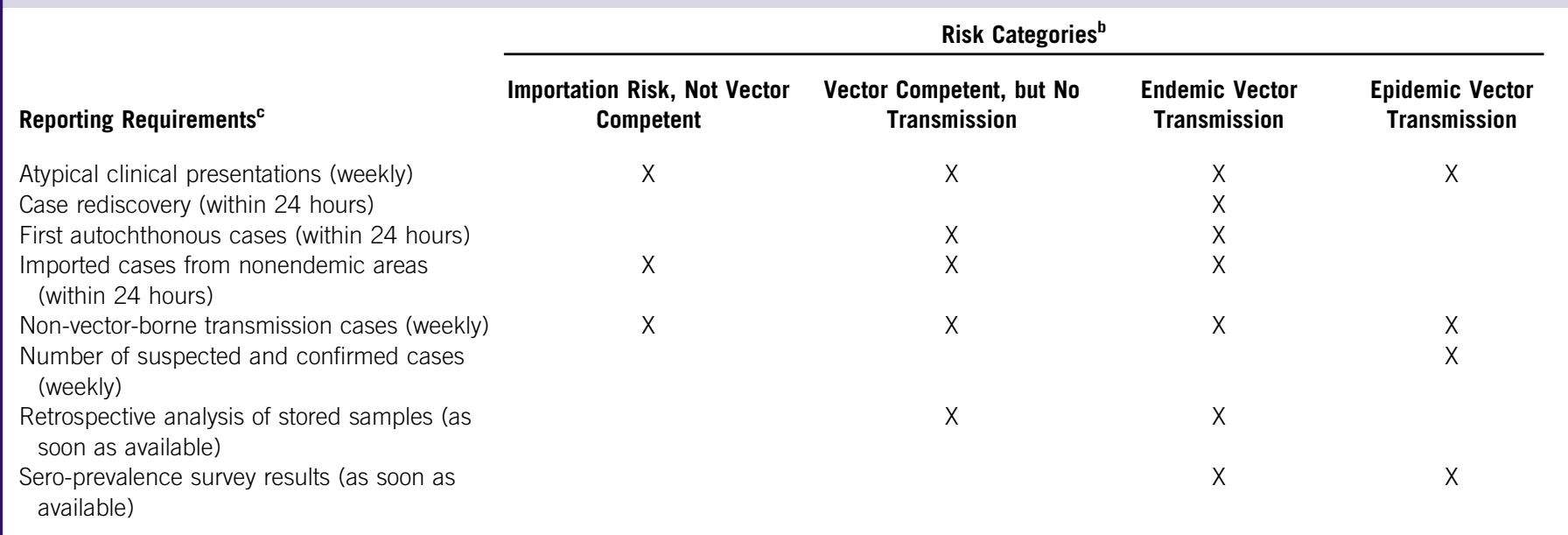

${ }^{a}$ Adapted from the World Health Organization Surveillance for Zika virus infection, microcephaly and Guillain-Barre syndrome: Interim Guidance (April 7, 2016). ${ }^{25}$ ${ }^{b}$ World Health Organization Zika virus surveillance risk categories.

${ }^{\mathrm{c}}$ Recommended reporting requirements. 
surveillance condition. The WHO's 4 transmission risk categories $^{25}$ are as follows:

1) "Countries with epidemic transmission of Zika virus" (ie, Brazil and French Polynesia) are those countries afflicted with sustained human outbreaks, characterized by "no evidence of circulation in the past," and "ongoing vector-borne transmission."

2) "Countries with possible endemic Zika virus transmission" include countries where transmission appears to occur at lower levels or occurred in the past.

3) "Countries at risk of Zika virus transmission" are countries with competent mosquito vectors but that have not experienced vector-borne Zika transmission. They are at risk of ZIKV introduction and further circulation through vector-borne transmission.

4) "Countries with no/low risk of mosquito-borne Zika virus transmission" are countries currently without competent mosquito vectors, based on existing data. They are at risk of case importation with the potential for further transmission through modes other than mosquitoes.

Recommended surveillance activities (Table 1) as well as key reporting requirements (Table 2 ) are provided based on the country's transmission risk or rating. By use of the same WHO guidance, Guillain-Barré and microcephaly surveillance and reporting would use the transmission risk categories and follow a similar pattern with a focus on each specific condition. Activities in "countries with epidemic transmission of Zika virus" would initially include recognition of serious cases presenting with each disease presentation, which would then trigger the initiation of additional surveillance procedures. As identified by Franca et al, however, added practices may be required for third-trimester fetal exposures as these babies may present with normocephalic heads with increased potential of brain abnormalities.

Conceptually, these transmission risk categories may be compared and matched to the WHO-identified phase descriptions and actions of a pandemic. ${ }^{26}$ The risk characterization system may be a useful approach that identifies specific triggers for scaling up (or down) surveillance and public health activities.

\section{PUBLIC HEALTH POLICY}

With ZIKV challenging medical and public health response within affected countries and threatening countries at risk of importation, public health authorities may need to revise their current disaster plans and response methodologies to address the specific issues raised by ZIKV. A sample model system is presented to define the level of engagement and the extent to which public health measures should be enacted. The degree to which public health responses need to be activated may depend on what "risk-impact zone" the country or geographic subunit (village, province, etc) falls into on the basis of the WHO ZIKV transmission risk categories compared with various disease impact areas (Table 3). Some initial considerations include the following:

1) How to collect, analyze, and report coordinated data from 2 contemporaneous but separate efforts (ie, disease surveillance and vector surveillance).

2) Knowledge gained from cross-disciplinary engagement by exercising the One Health concept.

3) How to display geographical distribution in an easily understood real-time format. This should be scalable

\section{TABLE 3}

Risk-Impact Zones Based on Zika Virus Disease Impact Areas Compared With Zika Virus Transmission Risk Categories: A Sample Model Approach

\begin{tabular}{|c|c|c|c|c|}
\hline \multirow[b]{2}{*}{ Impact Areas $^{b}$} & \multicolumn{4}{|c|}{ Risk Categories $^{a}$} \\
\hline & $\begin{array}{l}\text { Importation Risk, Not Vector } \\
\text { Competent }\end{array}$ & $\begin{array}{c}\text { Vector Competent, but No } \\
\text { Transmission }\end{array}$ & $\begin{array}{l}\text { Endemic Vector } \\
\text { Transmission }\end{array}$ & $\begin{array}{l}\text { Epidemic Vector } \\
\text { Transmission }\end{array}$ \\
\hline Condom availability & $-{ }^{c}$ & + & +++ & +++ \\
\hline Disease surveillance & + & ++ & +++ & ++++ \\
\hline Economic impact & - & - & +++ & ++++ \\
\hline Health care capacity & - & + & +++ & +++ \\
\hline $\begin{array}{l}\text { Need for enhanced } \\
\text { counseling }\end{array}$ & + & + & +++ & ++++ \\
\hline Need to revise plans & + & ++ & +++ & ++++ \\
\hline $\begin{array}{l}\text { Vector control/ } \\
\text { surveillance }\end{array}$ & - & ++ & +++ & ++++ \\
\hline
\end{tabular}

\footnotetext{
${ }^{a}$ World Health Organization Zika virus transmission risk categories. ${ }^{25}$

${ }^{\mathrm{b}}$ Disease impact areas.

${ }^{\mathrm{c}}$ Risk-impact zones defined: level; response action (-) normal; maintain public health measure; $(+)$ low; review and supplement public health measure; $(++)$ moderate; activate public health measure; $(+++)$ high; activate and expand public health measure; $(++++)$ extreme; activate, expand, and surge the public health measure.
} 
from the local to the national level, because A. aegypti generally spends its entire lifespan within a small radius of a few city blocks of its hatching; likewise, this is the justification for aggressive eradication control strategies around each infected case. ${ }^{27}$

4) How to educate and motivate respective populations pertaining to extended periods of condom use among vulnerable populations (ie, sexually active).

5) Planning for increased long-term health care and costs if there are expected increases in affected births or neurologic complications due to infection.

\section{CONCLUSION}

Although ZIKV has been studied for nearly 70 years, much remains to be discovered, because its viral attributes, disease epidemiology, and clinical characteristics are poorly defined. The ongoing ZIKV outbreak has proven dynamic and complex. The virus's status as a rapidly spreading, vectorborne, and mosquito-transmitted disease complicated by sexual transmission and neurophilic activity demands institution of key activities to affect change and control disease transmission.

Proactive and integrated vector control measures will be instrumental in reducing the adverse effects of ZIKV infection. Focused government financial and resource support along with increased local-level public health and environmental training and control efforts will be essential to reducing vector-borne transmission. Further vaccine development efforts appear promising but will take time to test and prepare for use. Yet questions will continue to remain as to vaccine effectiveness, longevity, and ability to protect both mother and fetus.

The WHO interim guidance for disease transmission and reporting provides a framework for deeper public health assessment. The use of this same outline and construct lends itself to our sample model approach to assessing public health response and activities that will assist in the decision-making process for this relatively new, complicated, and controversial public health issue.

\section{About the Authors}

Center for Global Health Engagement, Uniformed Services University of the Health Sciences, Rockville, Maryland.

Correspondence and reprint requests to Laura Singer, MPH, Research and Policy Analysist, Center for Global Health Engagement, Uniformed Services University of the Health Sciences, 11300 Rockville Pike, Suite 707, Rockville, MD 20852 (email: lsinger@cghe.org).

\section{Disclaimer}

The views expressed in this article are those of the authors and do not reflect the official policy or position of the Uniformed Services University of the Health Sciences, Department of Defense, or the US Government. The authors declare no competing interests.

Published online: August 30, 2016.

\section{REFERENCES}

1. Burkle FM. Zika: defining the public health and exposing its vulnerabilities. Disaster Med Public Health Prep. 2016;10(2):296-297. doi: $10.1017 / \mathrm{dmp} .2016 .27$.

2. World Health Organization. WHO statement on the first meeting of the International Health Regulations (2005) (IHR 2005) Emergency Committee on Zika virus and observed increase in neurological disorders and neonatal malformations. http://www.who.int/mediacentre/news/ statements/2016/1st-emergency-committee-zika/en/. Published February 1, 2016. Accessed February 2, 2016.

3. Brasil P, Sequeira PC, Freitas AD, et al. Guillain-Barre syndrome associated with Zika virus infection. Lancet. 2016;387(10026):1482. doi: 10.1016/S0140-6736(16)30058-7.

4. Broutet N, Krauer F, Riesen M, et al. Zika virus as a cause of neurologic disorders. N Engl J Med. 2016;374(16):1506-1509. doi: 10.1056/ NEJMp1602708.

5. Rasmussen SA, Jamieson DJ, Honein MA, et al. Zika virus and birth defects-reviewing the evidence for causality. N Engl J Med. 2016;374 (20):1981-1987. doi: 10.1056/NEJMsr1604338.

6. World Health Organization. International Health Regulations (2005), 2nd ed. Geneva: Switzerland; 2008:74.

7. Katz R, Fischer J. The Revised International Health Regulations: A Framework for Global Pandemic Response. Global Health Governance. 2010;III(2):1-18.

8. Fauci AS, Morens DM. Zika virus in the Americas-yet another arbovirus threat. N Engl J Med. 2016;374(7):601-604. doi: 10.1056/NEJMp1600297.

9. Larocca RA, Abbink P, Peron JP, et al. Vaccine protection against Zika virus from Brazil [published online June 28, 2016]. Nature. doi: 10.1038/ nature18952.

10. Naccache SN, Thézé J, Sardi I, et al. Discovery of a persistent Zika virus lineage in Bahia, Brazil [published online April 24, 2016]. bioRxiv. doi: $10.1101 / 049916$.

11. World Health Organization. WHO public health advice regarding the Olympics and Zika virus. http://www.who.int/mediacentre/news/state ments/2016/zika-health-advice-olympics/en/. Published May 28, 2016. Accessed June 8, 2016.

12. Grills A, Morrison S, Nelson B, et al. Projected Zika virus importation and subsequent ongoing transmission after travel to the 2016 Olympic and Paralympic Games-country-specific assessment, July 2016 [published online July 13. 2016]. MMWR Morb Mortal Wkly Rep. doi: http://dx.doi.org/10.15585/mmwr.mm16528e15581.

13. Kilpatrick AM, Randolph SE. Drivers, dynamics, and control of emerging vector-borne zoonotic diseases. Lancet. 2012;380(9857):1946-1955. doi: 10.1016/S0140-6736(12)61151-9.

14. Musso D, Cao-Lormeau VM, Gubler DJ. Zika virus: following the path of dengue and chikungunya? Lancet. 2015;386(9990):243-244. doi: 10.1016/S0140-6736(15)61273-9.

15. Araujo HR, Carvalho DO, Ioshino RS, et al. Aedes aegypti control strategies in Brazil: incorporation of new technologies to overcome the persistence of dengue epidemics. Insects. 2015;6(2):576-594. doi: 10.3390/ insects6020576.

16. Gubler DJ. Prevention and control of Aedes aegypti-borne diseases: lesson learned from past successes and failures. Asia Pac J Mol Biol Biotechnol. 2011;19(3):111-114.

17. Halstead SB. Successes and failures in dengue control-global experience. Dengue Bulletin. 2000;24:60-70.

18. Duchin JS. US public health preparedness for Zika and other threats remains vulnerable. Disaster Med Public Health Prep. 2016;10(2):298-299. doi: $10.1017 / \mathrm{dmp} .2016 .26$.

19. Paul LM, Carlin ER, Jenkins MM, et al. Dengue virus antibodies enhance Zika virus infection [published online April 25, 2016]. bioRxiv. doi: $10.1101 / 050112$.

20. Davidson A, Slavinski S, Komoto K, et al. Suspected female-to-male sexual transmission of Zika virus-New York City, 2016 [published online July 15, 2016]. MMWR Morb Mortal Wkly Rep. doi: http://dx.doi. org/10.15585/mmwr.mm16528e15582. 
21. Costello A, Dua T, Duran P, et al. Defining the syndrome associated with congenital Zika virus infection. Bull World Health Organ. 2016; 94(6):406-406A. doi: 10.2471/BLT.16.176990.

22. Rabe IB, Staples JE, Villanueva J, et al. Interim guidance for interpretation of Zika virus antibody test results [published online May 31, 2016]. MMWR Morb Mortal Wkly Rep. doi: http://dx.doi.org/ 2010.15585/mmwr.mm16521e15581.

23. World Health Organization. Early detection, assessment and response to acute public helath events: Implementation of Early Warning and Response with a focus on Event-Based Surveillance. http://www.who.int/ihr/publications/ WHO_HSE_GCR_LYO_2014.4/en/. Published 2014. Accessed July 8, 2016.

24. Franca GV, Schuler-Faccini L, Oliveira WK, et al. Congenital Zika virus syndrome in Brazil: a case series of the first 1501 livebirths with complete investigation [published online June 29, 2016]. Lancet. doi:10.1016/ S0140-6736(1016)30902-30903.

25. World Health Organization. Surveillance for Zika virus infection, microcephaly and Guillain-Barre syndrome: Interim guidance. http:// www.who.int/csr/resources/publications/zika/surveillance/en/. Published April 7, 2016. Accessed July 8, 2016.

26. World Health Organization. WHO Pandemic Phase Descriptions and Main Actions by Phase. http://www.who.int/influenza/resources/ documents/pandemic_phase_descriptions_and_actions.pdf. Published 2009. Accessed July 8, 2016.

27. McAllister J. Vector surveillance and control in reponse to Zika. https:// www.cdc.gov/zika/pdfs/zika-zapwebinar-5-17-16.pdf. Published May 25, 2016. Accessed July 8, 2016. 